\title{
ERRATUM
}

\section{Erratum to: A chemo-ecologists' practical guide to compositional data analysis}

\author{
Adrian Brückner ${ }^{1} \cdot$ Michael Heethoff $^{1}$
}

/ Published online: 8 December 2016

(C) Springer International Publishing 2016

\section{Erratum to: Chemoecology \\ DOI 10.1007/s00049-016-0227-8}

In the original publication of the article Table 4 was incorrect. The correct Table 4 is given below:
Table 4 Confusion matrices of a linear discriminant analysis (LDA) from (I) proportions and (II) with clr-transformed proportions

\begin{tabular}{lllccc}
\hline \multicolumn{2}{l}{ I: proportions } & & & & \\
\hline & LAR & PRO & DEU & TRI & ADU \\
\hline LAR & 5 & 0 & 0 & 0 & 0 \\
PRO & 0 & 6 & 0 & 0 & 0 \\
DEU & 0 & 0 & 19 & 0 & 0 \\
TRI & 0 & 1 & 0 & 17 & 0 \\
ADU & 0 & 0 & 0 & 0 & 20 \\
\hline II: clr-transformation & & & & \\
\hline LAR & 4 & 1 & 0 & 0 & 0 \\
PRO & 0 & 6 & 0 & 0 & 0 \\
DEU & 0 & 0 & 19 & 0 & 0 \\
TRI & 0 & 0 & 0 & 18 & 0 \\
ADU & 0 & 0 & 0 & 0 & 20
\end{tabular}

The online version of the original article can be found under doi:10.1007/s00049-016-0227-8.

Michael Heethoff

heethoff@bio.tu-darmstadt.de

Adrian Brückner

adrian.brueckner@gmail.com

1 Ecological Networks, Technische Universität Darmstadt,

Schnittspahnstraße 3, 64287 Darmstadt, Germany 